\title{
Management experiences of primary angiosarcoma of breast: a retrospective study from single institute in the People's Republic of China
}

This article was published in the following Dove Press journal:

OncoTargets and Therapy

4 November 2015

Number of times this article has been viewed

\author{
Qun-Chao $\mathrm{Hu}^{1-3, *}$ \\ Xin $\mathrm{Mei}^{1,2, *}$ \\ Yan Feng $g^{1,2}$ \\ Jin-Li Ma ${ }^{1,2}$ \\ Zhao-Zhi Yang ${ }^{1,2}$ \\ Zhi-Min Shao ${ }^{2,4}$ \\ Xiao-Li Yu ${ }^{1,2}$ \\ Xiao-Mao Guo',2
}

'Department of Radiation Oncology, Fudan University Shanghai Cancer

Center, ${ }^{2}$ Department of Oncology,

Shanghai Medical College, Fudan

University, Shanghai, ${ }^{3}$ Department of

Radiation Oncology, Suzhou Municipal

Hospital, Suzhou, ${ }^{4}$ Department of

Breast Surgery, Fudan University

Shanghai Cancer Center, Shanghai,

People's Republic of China

*These authors contributed equally to this work
Correspondence: Xiao-Li Yu; Xiao-Mao Guo

Department of Radiation Oncology, Fudan University Shanghai Cancer Center, 270 Dong An Road, Shanghai 200032, People's Republic of China Tel +86 2I 64175590 Fax +86 2I 64I74774 Email stephanieyxl@hotmail.com; guoxiaomaol88@outlook.com
Background: Primary angiosarcoma of breast (PAOB) is a rare and highly aggressive malignancy. There is no general agreement on optimal treatments or prognostic factors for this orphan disease. The objective of this study was to investigate the clinicopathologic features and management experiences of PAOB.

Methods: We performed a retrospective review of medical and pathologic records of 17 consecutive patients diagnosed with PAOB between January 2000 and February 2014 at FuDan University Shanghai Cancer Center. We evaluated the clinical characteristics, multimodality treatments, and associated clinical outcomes.

Results: A total of 16 patients were included in this retrospective study (median age at PAOB presentation 33.5 years, range: 19-56 years). Palpable tumor with or without breast skin ecchymosis presented as the most common initial symptom. All patients underwent surgery with curative intent. Median disease-free survival and overall survival (OS) were 9 months and 13.6 months, respectively. One-year and 3-year disease-free survival rates were $43.8 \%$ and $6.3 \%$, with OS rates of $93.8 \%$ and $78.1 \%$, respectively. High histologic grade indicated poorer OS by univariate analysis $(P=0.01)$. However, neither adjuvant chemotherapy nor radiotherapy contributed to clinical outcomes in our series.

Conclusion: PAOB is considered as an infrequent breast neoplasm with aggressive characteristics. Histologic grade and early metastasis (within 12 months after diagnosis) are associated with poor prognosis. Regardless of grade, additional benefit was not observed with adjuvant therapy.

Keywords: primary angiosarcoma, breast neoplasm, histologic grade, adjuvant therapy

\section{Introduction}

Angiosarcoma arises from endovascular tissue in various sites spontaneously or secondarily to ionizing radiation or chronic lymphedema. ${ }^{1}$ With the development of breast conservation therapy, secondary angiosarcoma (SA) seems somewhat increasing after the primary adjuvant radiotherapy (RT) and attracts extensive concern. ${ }^{2-4}$ Primary angiosarcoma of breast (PAOB) is also a comparatively rare type accounting for $<0.1 \%$ of malignant breast neoplasms. ${ }^{5}$ Previous comparisons of the two types of breast angiosarcoma did acknowledge the similarity in malignant behavior and poor prognosis. ${ }^{6,7}$ However, PAOB generally arises within the parenchyma and is considered rather histologically and clinically distinct from radiation-induced breast angiosarcoma.

PAOB usually presents painless mass as the initial symptom, occasionally with skin involvement. It shows poorer outcome than other histologic types of primary breast sarcomas. ${ }^{8}$ Earlier studies report that the 5 -year overall survival (OS) rate varied from 
$40 \%$ to $85 \%$. ${ }^{7,9}$ Surgical resection remains the most important curative treatment for localized PAOB. In addition, the role of adjuvant chemotherapy and/or RT has also been elucidated, which suggested that patients with high risk of recurrence might benefit from adjuvant therapy. ${ }^{10,11}$

Typically, tumor size, pathologic grade, and margin status are commonly accepted as important factors that may potentially affect OS in other soft tissue sarcomas. Mastectomy with clear margin is the most frequent choice in the operable patients with a large tumor. However, there are still controversial reports in the literature regarding histologic grade as a prognostic factor guiding adjuvant therapy. ${ }^{12,13}$

Due to the extremely low incidence of PAOB, evidence regarding the preferable management strategies or prognostic factors is limited. Therefore, we reviewed our institutional experience with PAOB to describe the clinical features and explore the impact of treatment modalities on disease-free survival (DFS) and OS.

\section{Materials and methods}

This retrospective study included 17 consecutive patients with PAOB treated with surgical resection between January 8, 2000, and February 18, 2014, at FuDan University Shanghai Cancer Center. One patient confirmed with invasive ductal breast cancer on the contralateral side was excluded for potential bias. This investigation was approved by the institutional review board of FuDan University Shanghai Cancer Center. Our institutional review board routinely obtains the initial consents from patients receiving treatments in our cancer center and we would inform the participants of present study again through calls or mails during the follow up. Medical and pathologic records of every patient were reviewed to verify the following information: family history of cancer and concomitant cancer, pathologic diagnosis, age at diagnosis, surgical margin status, primary tumor size, axillary lymph node status, and treatment modalities. Follow-up observations occurred until the patient died or the end of the study on April 17, 2015.

All patients underwent surgery with curative intent, and axillary dissection was performed according to individual situation. Axillary lymph node and vascular invasion status were not included in the analysis because of the low incidence of these events $(1 / 16,6.25 \%)$. Tumor was defined according to the World Health Organization Classification of Tumours of Soft Tissue and Bone, fourth edition. ${ }^{14}$ The cutoff value of tumor size was defined as $5 \mathrm{~cm}$ in diameter according to soft tissue sarcoma. The histologic grade of each case was assessed according to Rosen's three-tier system. The three grades approximately represent the differentiation and proliferation of the tumors. A tumor with low grade is observed with relatively well-structured tissue, whereas grade 3 tumor usually demonstrates features of highly proliferating malignant cells and intralesional necrosis. Negative margin status was reported as $\mathrm{R} 0$ for no residual tumor and $\mathrm{R} 1$ for microscopic residual tumor $<1 \mathrm{~mm}$ from the margin. Details on the levels of hormone receptors (estrogen receptor and progesterone receptor) and human epidermal growth factor receptor 2 were not included due to the low incidence of PAOB.

Adjuvant external-beam RT was delivered in five patients with $6 \mathrm{MV}$ X-ray beam. The most frequent RT dose was $56 \mathrm{~Gy}$ in 28 fractions. One patient received salvage RT after local recurrence (R1 resection), followed by administration of $50 \mathrm{~Gy}$ in 25 fractions and tumor bed boost of $10 \mathrm{~Gy}$. Seven patients received adjuvant chemotherapy, in which the most common regimen consisted of cyclophosphamide and doxorubicin. No neoadjuvant therapy was applied before surgery.

DFS and OS were assessed. DFS was defined as the time from the date of initial diagnosis to either local recurrence or distant metastasis. All the local recurrences were verified by biopsy, whereas distant metastases were mostly diagnosed clinically by imaging examinations. OS was calculated from the date of initial diagnosis to the time of death or the length of follow-up. For all survival analyses, the patients were censored, if no local recurrence, and distant metastasis was detected at the last follow-up or on the date of death. KaplanMeier method was used to estimate survival, while the log-rank test was used to perform curve comparison. Cox regression model was applied to determine the independent effect after adjusting for critical clinical features and treatment modalities. Statistical analyses were performed using SPSS, Version 11.0. Two-sided $P<0.05$ was considered statistically significant.

\section{Results}

The median age at diagnosis of the 16 female patients was 33.5 years (range: 19-56 years). Palpable tumor was the most frequent symptom at first diagnosis (10/16), and three patients complained of bruise on the breast skin with or without local edema. The median size of the tumor in the greatest diameter was $6 \mathrm{~cm}$ (range: $1.5-14 \mathrm{~cm}$ ). PAOB was confirmed as the only primary malignancy in all cases. No radiation or plastic surgery history was reported in any patients. The clinicopathologic features are summarized in Table 1.

Median follow-up period for the whole group was 45 months (range, 8-129 months). During the analysis, six patients had developed local recurrence and five of them were found to have had a relapse near the tumor bed. Only one 
Table I Clinicopathologic characteristics and treatment modalities of 16 patients with $\mathrm{PAOB}$

\begin{tabular}{|c|c|c|}
\hline Characteristics & $\begin{array}{l}\text { Number } \\
\text { of patients }\end{array}$ & n (\%) \\
\hline Patient age (years), median (ranges) & $33.5(19-56)$ & \\
\hline Follow-up time (months), median (ranges) & $45(8-129)$ & \\
\hline \multicolumn{3}{|l|}{ Menopausal status } \\
\hline Premenopausal & 13 & 81.2 \\
\hline Postmenopausal & 3 & 18.8 \\
\hline \multicolumn{3}{|l|}{ Size of tumor $(\mathrm{cm})$} \\
\hline$\leq 5$ & 13 & 81.2 \\
\hline$>5$ & 3 & 18.8 \\
\hline \multicolumn{3}{|l|}{ Histologic grade } \\
\hline Grade I & 8 & 50 \\
\hline Grade 2 & 5 & 31.2 \\
\hline Grade 3 & 3 & 18.8 \\
\hline \multicolumn{3}{|l|}{ Initial surgical procedure } \\
\hline Lumpectomy & 3 & 18.8 \\
\hline Mastectomy & 13 & 81.2 \\
\hline \multicolumn{3}{|l|}{ Axillary dissection } \\
\hline Yes & 3 & 18.8 \\
\hline No & 13 & 81.2 \\
\hline \multicolumn{3}{|l|}{ Adjuvant chemotherapy } \\
\hline Yes & 7 & 43.8 \\
\hline No & 9 & 56.2 \\
\hline \multicolumn{3}{|l|}{ Adjuvant radiotherapy } \\
\hline Yes & 5 & 31.2 \\
\hline No & II & 68.8 \\
\hline
\end{tabular}

Abbreviations: PAOB, primary angiosarcoma of breast; $n$, number.

patient appeared to have had a multifocal ipsilateral breast recurrence. All patients with local or regional recurrences underwent salvage surgery. Three patients were confirmed with $\mathrm{R} 0$ resection, while two patients were of close margin $(>1 \mathrm{~mm})$ and another one of $\mathrm{R} 1$ resection. Metastatic tumor was confirmed in 14 patients during the follow-up. Five of them occurred within the first 12 months after diagnosis and presented poorer outcomes than those over 12 months $(P=0.003$; Figure 1$)$. The first sites of solitary metastasis were reported as the bone (5/14), lung (2/14), soft tissue of limbs (2/14), and skin beyond the ipsilateral breast (2/14). Multisite metastases occurred initially in other five patients. There was no significant difference in the clinical survival between different metastatic sites.

As shown in Figure 2, median DFS was 9 months (95\% CI 3.1-14.9 months) with 1-year DFS of $43.8 \%$ and 3-year DFS of $6.3 \%$, while median OS was 13.6 months $(95 \% \mathrm{CI}$ 8.8-44.1 months) with 1-year OS of $93.8 \%$ and 3-year OS of $78.1 \%$. Table 2 summarizes the results of univariate risk factor analysis for the whole entity by tumor characteristics and treatment modalities. Because of the limited sample size of the series and the lower proportion of large tumor size, we found no significant differences in the univariate analysis of OS in terms of tumor size $(P=0.104)$, initial surgical procedure $(P=0.321)$,

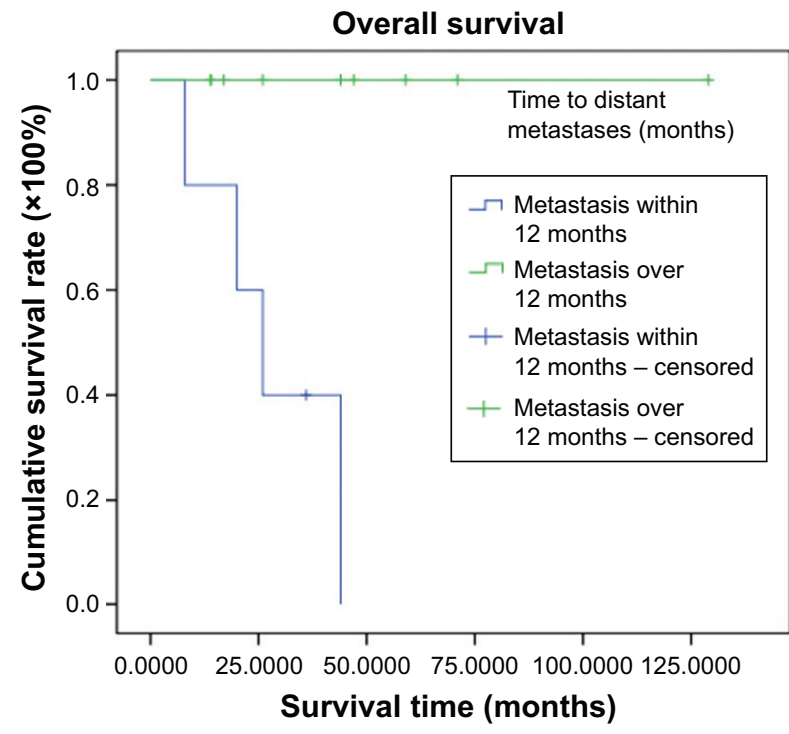

Figure I Kaplan-Meier curves of overall survival (OS) of 14 metastasis patients with primary angiosarcoma of breast.

Note: Early metastasis (within 12 months after diagnosis) is associated with poor OS $(P=0.003)$.

adjuvant chemotherapy ( $P=0.913)$, or RT ( $P=0.484)$. Only high histologic grade was found to be associated with decreased DFS and OS ( $P=0.047$ for DFS and $P=0.001$ for OS). DFS and $\mathrm{OS}$ rates in relation to histologic grade are illustrated in Figure 3. Since all patients with pathologic grade 1 confirmed of survival status were censored, the estimated OS can be expected in a longer follow-up. As shown in Figure 4, compared to the sheets of tumor cells in grade 3 lesions, PAOB with grade 1 was composed of relatively well-formed structure permeated with fat and normal vascular channels.

All the patients achieved clear margin after initial surgery, and there was no difference between lumpectomy and mastectomy. Axillary dissection was not mandatory and was performed in only three patients. Therefore, only the effect of adjuvant chemotherapy and RT on DFS and OS was considered together with histologic grade in the multivariate analysis (Table 3). Nevertheless, there was no independent prognostic factor confirmed in the present study, including histologic grade ( $P=0.491$ for DFS and $P=0.464$ for OS).

\section{Discussion}

Breast angiosarcoma is a very rare and invasive disease, which comprises $<0.08 \%$ of breast malignancies in our institution. SA is a common form of angiosarcoma that is usually induced by irradiation or chronic lymphedema (Stewart-Treves syndrome) and has been much discussed in descriptive studies and population-based analysis. ${ }^{2,9,15,16}$ A previous study showed that $\mathrm{PAOB}$ exhibited different 
A

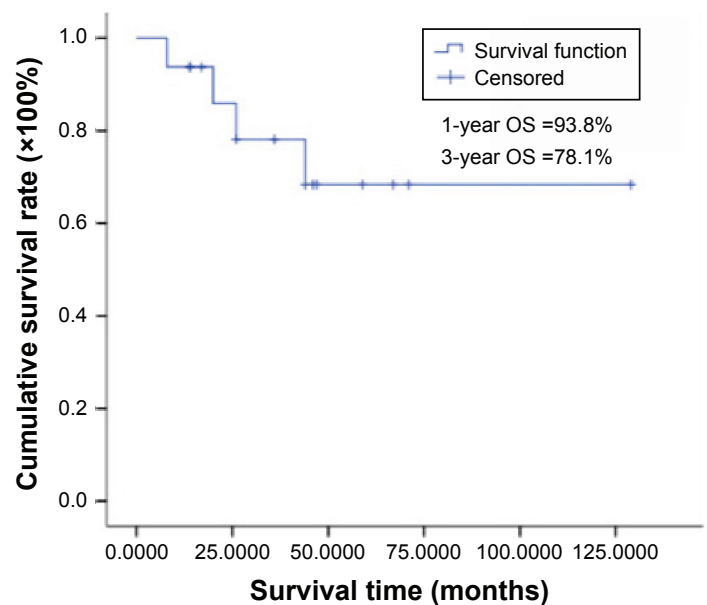

B

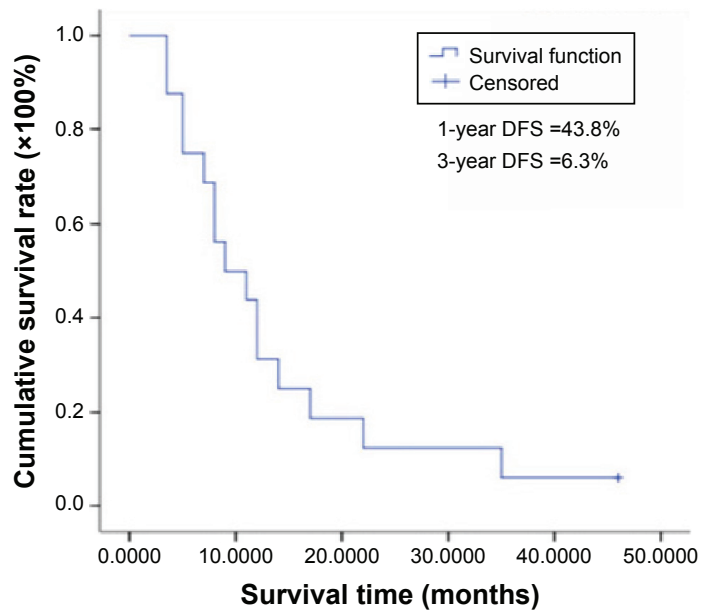

Figure 2 Kaplan-Meier curves of overall survival (A) and disease-free survival (B) of 16 patients with primary angiosarcoma of breast. Abbreviations: DFS, disease-free survival; OS, overall survival.

clinical and pathologic features compared with SA. ${ }^{17}$ However, few studies have focused on this type of angiosarcoma. In our series, all the patients declared no radiation history and were histologically confirmed as PAOB.

The median age of our patients with $\mathrm{PAOB}$ was 33.5 years, which indicate a younger age than that of $\mathrm{PAOB}$ patients reported in Western countries. ${ }^{6}$ However, young age might not be an unfavorable factor regarding to OS in
$\mathrm{PAOB}$, which is different from invasive ductal carcinoma of the breast. All patients except one developed recurrence or metastases, and four patients died of PAOB. Three-year DFS and OS rates were $6.3 \%$ and $78.1 \%$, respectively. A high proportion $(87.5 \%)$ of metastases resulted in an inferior DFS, which is comparable with previous studies. ${ }^{18,19}$ However, it was the time to metastasis and not the metastasis status or sites that ultimately affected the OS. Five patients (31.3\%)

Table 2 Univariate analysis of factors associated with DFS and OS of PAOB

\begin{tabular}{|c|c|c|c|c|c|}
\hline \multirow[t]{2}{*}{ Characteristics } & \multirow{2}{*}{$\begin{array}{l}\text { Number } \\
\text { of patients }\end{array}$} & \multicolumn{2}{|l|}{ DFS } & \multicolumn{2}{|l|}{ OS } \\
\hline & & $\begin{array}{l}\text { Number of events } \\
\text { ( } 3 \text { years of survival \%) }\end{array}$ & $\overline{P \text {-value }}$ & $\begin{array}{l}\text { Number of events } \\
\text { ( } 3 \text { years of survival \%) }\end{array}$ & $P$-value \\
\hline Age (years) & & & 0.976 & & 0.49 \\
\hline$\leq 50$ & 14 & $13(92.9)$ & & $4(28.6)$ & \\
\hline$>5$ & 2 & $2(100)$ & & NE & \\
\hline Tumor size $(\mathrm{cm})$ & & & 0.869 & & 0.104 \\
\hline$\leq 5$ & 13 & $12(92.3)$ & & $2(15.4)$ & \\
\hline$>5$ & 3 & $3(100)$ & & $2(66.7)$ & \\
\hline Histologic grade & & & 0.047 & & 0.001 \\
\hline Grade I & 8 & 7 (87.5) & & NE & \\
\hline Grade 2 & 5 & $5(100)$ & & I (20) & \\
\hline Grade 3 & 3 & $3(100)$ & & $3(100)$ & \\
\hline Initial surgical procedure & & & 0.822 & & 0.321 \\
\hline Lumpectomy & 3 & $3(100)$ & & NE & \\
\hline Mastectomy & 13 & $12(92.3)$ & & $4(30.8)$ & \\
\hline Axillary dissection & & & 0.496 & & 0.429 \\
\hline Yes & 3 & $3(100)$ & & I (33.3) & \\
\hline No & 13 & $12(92.3)$ & & $3(23.1)$ & \\
\hline Adjuvant chemotherapy & & & 0.419 & & 0.913 \\
\hline Yes & 7 & $7(100)$ & & $2(28.6)$ & \\
\hline No & 9 & $8(88.9)$ & & $2(22.2)$ & \\
\hline Adjuvant radiotherapy & & & 0.167 & & 0.484 \\
\hline Yes & 5 & $5(100)$ & & I (20) & \\
\hline No & 11 & $10(90.9)$ & & $3(27.3)$ & \\
\hline Salvage surgery after progress & & & & & 0.13 \\
\hline Yes & 6 & & & $3(20)$ & \\
\hline No & 10 & & & $\mathrm{I}(10)$ & \\
\hline
\end{tabular}

Abbreviations: DFS, disease-free survival; OS, overall survival; PAOB, primary angiosarcoma of breast; NE, no event. 
A

Overall survival

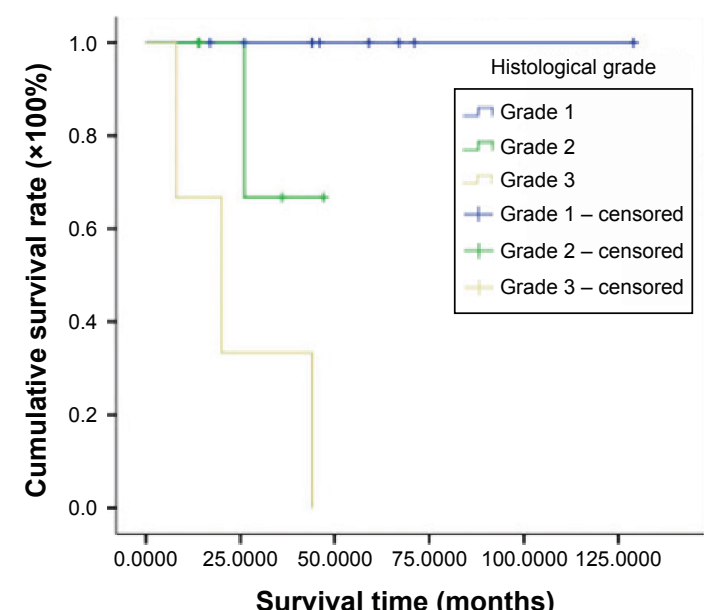

B

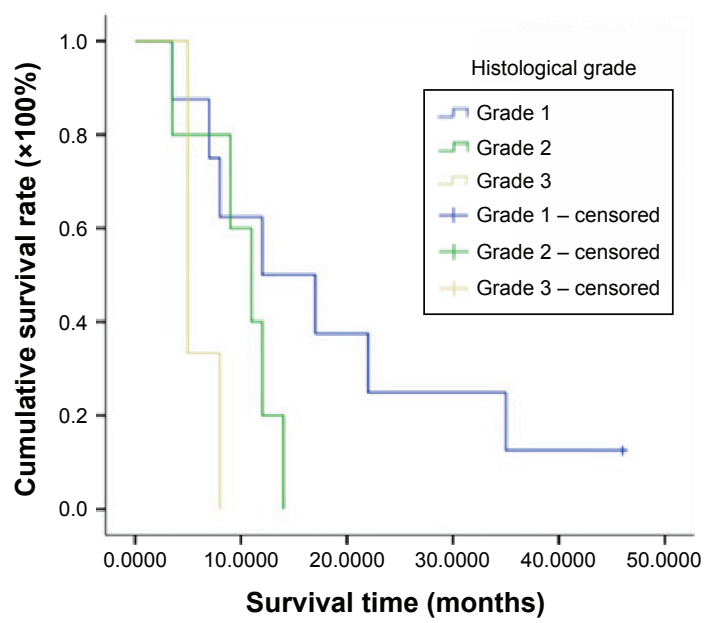

Figure 3 Overall survival (OS) (A) and disease-free survival (DFS) (B) by histologic grade in patients with primary angiosarcoma of breast. Note: High histologic grade indicated poorer prognosis for both OS $(P=0.001)$ and DFS $(P=0.047)$.

with early metastasis that occurred within 12 months after diagnosis presented worse outcomes $(P=0.003)$. Since a significant difference was hardly confirmed due to the small sample size of the series, we descriptively found that most of the early metastasis occurred in patients with large tumor size, poor differentiation, and non-R0 resection after salvage surgery. Because all patients achieved clear surgical margin in the initial resection including those with great primary tumor burden, it seems difficult to properly evaluate the effect of tumor size or margin status on distant metastasis. Although wide excision margin of at least $5 \mathrm{~cm}$ has been recommended for better recurrence-free and survival rate,,$^{20}$ aggressive surgical approach might be impractical in large masses, especially in salvage surgery. Furthermore, positive margins were considered to be associated with higher risk of local recurrence but not always relative to poorer OS. ${ }^{21}$

High histologic grade of $\mathrm{PAOB}$ was frequently reported to be an unfavorable prognostic factor in retrospective series
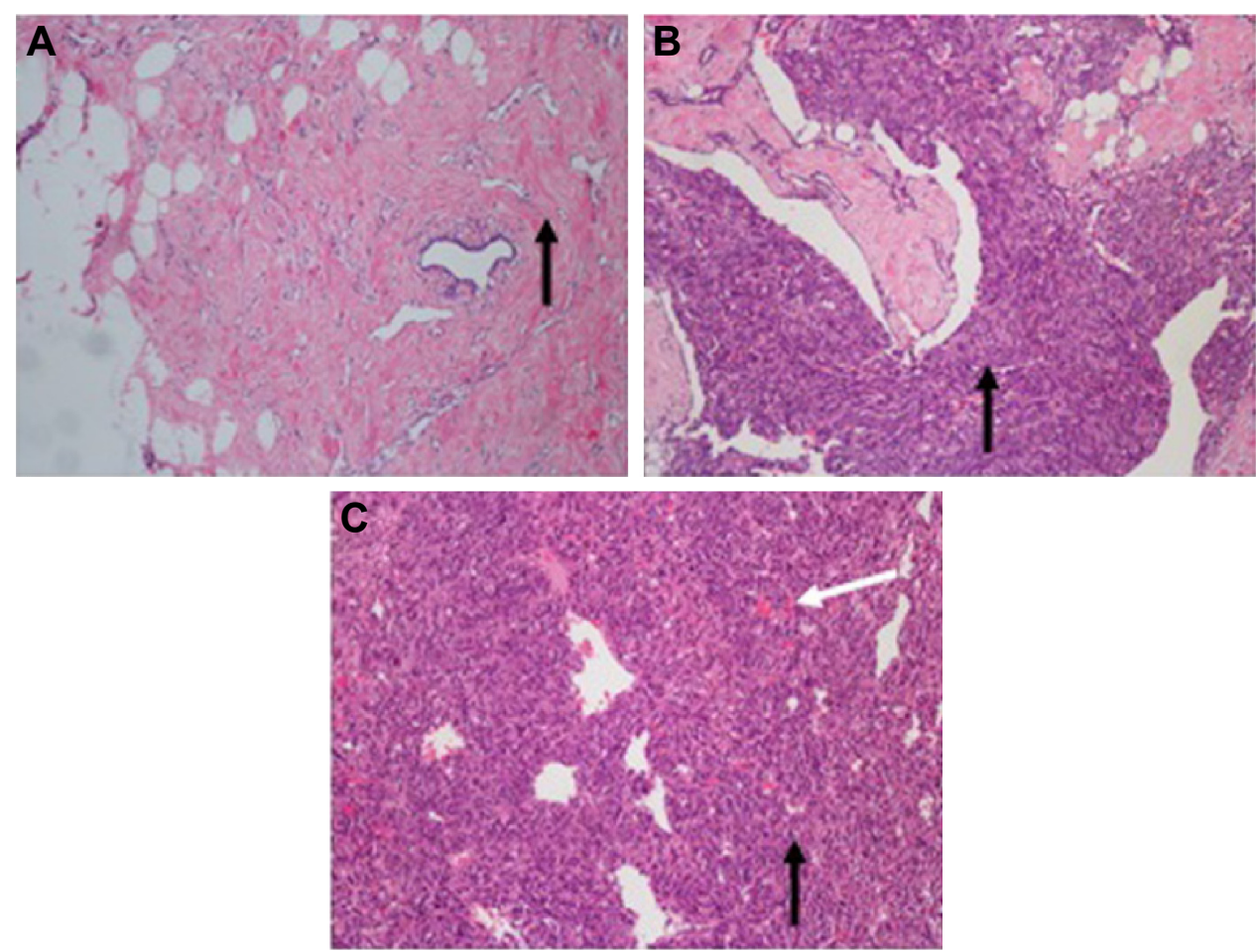

Figure 4 Hematoxylin-eosin-stained sections of primary angiosarcoma of breast (magnification $\times 100$ ).

Notes: Grade I tumor lesion (A) scattered with varied shapes of malignant cells (black arrow) and normal capillary vessel. Grade 2 tumor lesion (B) with multilayer cells with distinct nuclear atypia. Grade 3 tumor lesion $(\mathbf{C})$ composed of sheets of high nuclear grade cells with hemorrhagic necrosis sites (white arrow). 
Table 3 Multivariate analysis of factors associated with DFS and OS of PAOB

\begin{tabular}{|c|c|c|c|c|}
\hline \multirow[t]{2}{*}{ Characteristics } & \multicolumn{2}{|l|}{ DFS } & \multicolumn{2}{|l|}{ OS } \\
\hline & $P$-value & HR (95\% CI) & $P$-value & HR (95\% CI) \\
\hline Histologic grade & 0.491 & $\mathrm{I} .5(0.5-5.2)$ & 0.464 & $67.3(0.01-5,333,118)$ \\
\hline Adjuvant chemotherapy & 0.175 & $2.2(0.7-6.7)$ & 0.980 & I.I (0-48,779.I) \\
\hline Adjuvant radiotherapy & 0.614 & $\mathrm{I} .4(0.4-5.1)$ & 0.247 & $0.001(0-101.2)$ \\
\hline
\end{tabular}

Abbreviations: DFS, disease-free survival; OS, overall survival; $\mathrm{PAOB}$, primary angiosarcoma of breast; $\mathrm{HR}$, hazard ratio; $\mathrm{Cl}$, confidence interval.

and population-based studies. ${ }^{13,18,22}$ As described by Rosen et al, ${ }^{23} 5$-year DFS was $76 \%$ in grade 1 tumor and dramatically fell to $15 \%$ in grade 3 tumors. Consistently, our findings indicated significantly better survival in patients with low histologic grade, as shown in Table 2. All patients with grade 3 tumors developed metastases within 12 months after initial treatment. Despite superior DFS and OS in grade 1 and grade 2, histologic grade was not recognized as an independent prognostic factor in the multivariate analysis. It might be due to the small number of high-grade patients and relatively short follow-up period. Indeed, histologic grade as a powerful prognostic factor is still controversial. Experience from Mayo Clinic showed tumor size rather than tumor grade as a more valuable prognostic factor. But there were only six patients diagnosed with angiosarcoma, and the authors particularly noted that the finding might be related to the small series of cases and limited statistical power. ${ }^{24}$ Similarly, in a systematic review of 74 articles, including both $\mathrm{PAOB}$ and $\mathrm{SA}$, tumor size and age rather than histologic grade were significant prognostic factors for local recurrence-free interval and OS. ${ }^{25}$ It should be noted that patients of advanced age and tumor size were collected in the analysis. Majority of patients (68\%) received surgery alone, while less proportion of the entity underwent surgery with RT $(17 \%)$ or chemotherapy $(6 \%)$. Thus, we must be cautious in the interpretation of these results.

Patients with PAOB are associated with poorer prognosis compared to patients with invasive ductal carcinomas or other breast sarcomas as a whole. Due to the rarity of the disease, there is no general agreement or specific guideline for $\mathrm{PAOB}$, and treatment remains a great challenge in clinical practice. Surgical resection still plays a predominant role in multimodality management strategies, but the optimal procedure is still under discussion. Kaklamanos et al supported a preferred effect with mastectomy over local resection. ${ }^{5}$ In our series, patients with tumor size $>3 \mathrm{~cm}$ in diameter and with high-grade disease chose to undergo mastectomy. Limited cases underwent local excision, but no significant difference was found between the two surgical approaches. As for the adjuvant therapy, including chemotherapy and RT, the clinical benefit varied in different reports and no definite advantages were found. ${ }^{12,13,25-27}$ Chemotherapy regimens were mostly extrapolated from soft tissue sarcomas of different origins. Target therapies, such as antiangiogenesis agents or molecular inhibitors, were investigated in only selective patients, as described in case reports or series. ${ }^{28,29}$ In clinical practice, large tumor size, high histologic grade, vascular invasion, and positive lymph nodes were generally accepted as high-risk factors that predicted the high recurrence and worse survival. In the present study, patients with these highrisk factors had a strong tendency to receive adjuvant chemotherapy and/or RT, and RT was routinely recommended for patients after lumpectomy. Since the PAOB might have similar low radiosensitivity as soft-tissue sarcoma in other sites, doses of $56 \mathrm{~Gy}$ for clear margin and 60Gy at least for microscopic positive margin after surgery were prescribed. However, we demonstrated that no additional benefit was found either with chemotherapy or with RT. It might be partially relative to the small size of the series and distinct biological behavior of the tumor in different populations or races.

Our study had several limitations. First of all, the present interpretation of results was based on a case series in a single institute. The small size of the sample might reduce the statistical power with regard to histologic grade as a prognostic marker. Second, our study was limited to a retrospective research; thus, inevitable bias might have been generated due to lack of a preestablished criterion to balance the intergroup distribution, incomplete information, and individualized treatments. Due to the less number of patients with tumor size $>5 \mathrm{~cm}$ and the high proportion of clear surgical margin, our findings should be interpreted with caution for cases with high-risk factors. Meanwhile, limited tissue samples restricted further exploration into biological behavior based on molecular markers. Despite these limitations, our study demonstrated the impact of histologic grade in PAOB and adjuvant therapy as well.

\section{Conclusion}

PAOB is an extremely rare and invasive tumor in all breast neoplasms. Surgical resection with safe margin remains the priority in curative treatment option. High histologic grade and early metastasis (within 12 months after diagnosis) were 
found to be associated with poor prognosis. Unfortunately, current adjuvant treatment options are limited. Neither adjuvant chemotherapy nor RT was found to have any advantage in clinical outcomes. Recent investigations tried to investigate target therapy associated with aberrant molecular or gene changes in PAOB, which might offer hope for a specific target therapy in future.

\section{Acknowledgment}

This work was partly supported by the National Natural Science Foundation of China (grant numbers 81372430 and 81402525).

\section{Disclosure}

The authors report no conflicts of interest in this work.

\section{References}

1. Young RJ, Brown NJ, Reed MW, Hughes D, Woll PJ. Angiosarcoma. Lancet Oncol. 2010;11:983-991.

2. Mery CM, George S, Bertagnolli MM, Raut CP. Secondary sarcomas after radiotherapy for breast cancer: sustained risk and poor survival. Cancer. 2009;115:4055-4063.

3. D'Angelo SP, Antonescu CR, Kuk D, et al. High-risk features in radiation-associated breast angiosarcomas. Br J Cancer. 2013;109: 2340-2346.

4. Morgan EA, Kozono DE, Wang Q, et al. Cutaneous radiation-associated angiosarcoma of the breast: poor prognosis in a rare secondary malignancy. Ann Surg Oncol. 2012;19:3801-3808.

5. Kaklamanos IG, Birbas K, Syrigos KN, Vlachodimitropoulos D, Goutas N, Bonatsos G. Breast angiosarcoma that is not related to radiation exposure: a comprehensive review of the literature. Surg Today. 2011;41: 163-168.

6. Arora TK, Terracina KP, Soong J, Idowu MO, Takabe K. Primary and secondary angiosarcoma of the breast. Gland Surg. 2014;3:28-34.

7. Hui A, Henderson M, Speakman D, Skandarajah A. Angiosarcoma of the breast: a difficult surgical challenge. Breast. 2012;21:584-589.

8. Zelek L, Llombart-Cussac A, Terrier P, et al. Prognostic factors in primary breast sarcomas: a series of patients with long-term follow-up. J Clin Oncol. 2003;21:2583-2588.

9. Luini A, Gatti G, Diaz J, et al. Angiosarcoma of the breast: the experience of the European Institute of Oncology and a review of the literature. Breast Cancer Res Treat. 2007;105:81-85.

10. Bhosale SJ, Kshirsagar AY, Patil MV, Wader JV, Nangare N, Patil PP Primary angiosarcoma of breast: a case report. Int J Surg Case Rep. 2013;4:362-364.

11. Ghareeb ER, Bhargava R, Vargo JA, Florea AV, Beriwal S. Primary and radiation-induced breast angiosarcoma clinicopathologic predictors of outcomes and the impact of adjuvant radiation therapy Am J Clin Oncol. 2014. Epub ahead of print.

OncoTargets and Therapy

\section{Publish your work in this journal}

OncoTargets and Therapy is an international, peer-reviewed, open access journal focusing on the pathological basis of all cancers, potential targets for therapy and treatment protocols employed to improve the management of cancer patients. The journal also focuses on the impact of management programs and new therapeutic agents and protocols on
12. Sher T, Hennessy BT, Valero V, et al. Primary angiosarcomas of the breast. Cancer. 2007;110:173-178.

13. Pandey M, Sutton GR, Giri S, Martin MG. Grade and prognosis in localized primary angiosarcoma. Clin Breast Cancer. 2015;15(4): 266-269.

14. Fletcher CDM, Bridge JA, Hogendoorn P, Mertens F. WHO Classification of Tumours of Soft Tissue and Bone. 4th ed. Geneva: International Agency for Research on Cancer; 2013:1-468.

15. Brenn T, Fletcher CD. Radiation-associated cutaneous atypical vascular lesions and angiosarcoma: clinicopathologic analysis of 42 cases. Am J Surg Pathol. 2005;29:983-996.

16. Gambini D, Visintin R, Locatelli E, et al. Secondary breast angiosarcoma and paclitaxel-dependent prolonged disease control: report of two cases and review of the literature. Tumori. 2015;101:e60-e63.

17. Fraga-Guedes C, Gobbi H, Mastropasqua MG, Botteri E, Luini A, Viale G. Primary and secondary angiosarcomas of the breast: a single institution experience. Breast Cancer Res Treat. 2012;132:1081-1088.

18. Bousquet G, Confavreux C, Magné N, et al. Outcome and prognostic factors in breast sarcoma: a multicenter study from the rare cancer network. Radiother Oncol. 2007;85:355-361.

19. Vorburger SA, Xing Y, Hunt KK, et al. Angiosarcoma of the breast. Cancer. 2005;104:2682-2688.

20. Benevento R, Carafa F, Di Nardo D. Angiosarcoma of the breast: a new therapeutic approach? Int J Surg Case Rep. 2015;13:30-32.

21. Barrow BJ, Janjan NA, Gutman H, et al. Role of radiotherapy in sarcoma of the breast-a retrospective review of the M.D. Anderson experience. Radiother Oncol. 1999;52:173-178.

22. Scow JS, Reynolds CA, Degnim AC, Petersen IA, Jakub JW, Boughey JC. Primary and secondary angiosarcoma of the breast: the Mayo Clinic experience. J Surg Oncol. 2010;101:401-407.

23. Rosen PP, Kimmel M, Ernsberger D. Mammary angiosarcoma. The prognostic significance of tumor differentiation. Cancer. 1988;62: 2145-2151.

24. Adem C, Reynolds C, Ingle JN, Nascimento AG. Primary breast sarcoma: clinicopathologic series from the Mayo Clinic and review of the literature. Br J Cancer. 2004;91:237-241.

25. Depla AL, Scharloo-Karels CH, de Jong MA, et al. Treatment and prognostic factors of radiation-associated angiosarcoma (RAAS) after primary breast cancer: a systematic review. Eur J Cancer. 2014;50: $1779-1788$.

26. Silverman LR, Deligdisch L, Mandeli J, Greenspan EM. Chemotherapy for angiosarcoma of the breast: case report of 30-year survival and analysis of the literature. Cancer Invest. 1994;12:145-155.

27. Fury MG, Antonescu CR, Van Zee KJ, Brennan MF, Maki RG A 14-year retrospective review of angiosarcoma: clinical characteristics, prognostic factors, and treatment outcomes with surgery and chemotherapy. Cancer J. 2005;11:241-247.

28. Silva E, Gatalica Z, Vranic S, Basu G, Reddy SK, Voss A. Refractory anigosarcoma of the breast with VEGFR2 upregulation successfully treated with sunitibib. Breast J. 2015;21:205-207.

29. Abbott R, Palmieri C. Angiosarcoma of the breast following surgery and radiotherapy for breast cancer. Nat Clin Pract Oncol. 2008;5: 727-736.

patient perspectives such as quality of life, adherence and satisfaction The manuscript management system is completely online and includes a very quick and fair peer-review system, which is all easy to use. Visit http://www.dovepress.com/testimonials.php to read real quotes from published authors. 